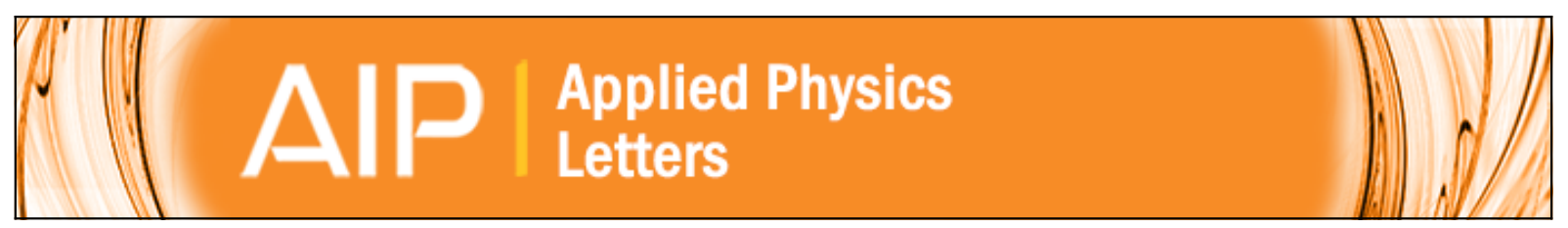

\title{
Theoretical and experimental investigations of asymmetric light transport in graded index photonic crystal waveguides
}

I. H. Giden, D. Yilmaz, M. Turduev, H. Kurt, E. Çolak, and E. Ozbay

Citation: Applied Physics Letters 104, 031116 (2014); doi: 10.1063/1.4861926

View online: http://dx.doi.org/10.1063/1.4861926

View Table of Contents: http://scitation.aip.org/content/aip/journal/apl/104/3?ver=pdfcov

Published by the AIP Publishing

\section{Articles you may be interested in}

Design and experimental evidence of a flat graded-index photonic crystal lens

J. Appl. Phys. 114, 083105 (2013); 10.1063/1.4817368

Gyrotropic photonic crystal waveguide switches

Appl. Phys. Lett. 96, 051125 (2010); 10.1063/1.3309715

Experimental demonstration of Bloch mode parity change in photonic crystal waveguide

Appl. Phys. Lett. 85, 2682 (2004); 10.1063/1.1795370

Enhancement of magneto-optical transverse electric-transverse magnetic mode conversion efficiency of $(\mathrm{Cd}, \mathrm{Mn}) \mathrm{Te}$ waveguide by graded-index clad layers

J. Appl. Phys. 95, 7181 (2004); 10.1063/1.1668571

Electrooptic Large Index Contrast Waveguides For LightWave Signal Processing

AIP Conf. Proc. 709, 91 (2004); 10.1063/1.1764015

\section{A.P $\left.\right|_{\text {Applied Physics }} ^{\text {Journal of }}$}

Journal of Applied Physics is pleased to announce André Anders as its new Editor-in-Chief 


\title{
Theoretical and experimental investigations of asymmetric light transport in graded index photonic crystal waveguides
}

\author{
I. H. Giden, ${ }^{1}{ }^{1, a)}$ D. Yilmaz, ${ }^{1}$ M. Turduev,${ }^{1}$ H. Kurt, ${ }^{1}$ E. Çolak, ${ }^{2}$ and E. Ozbay ${ }^{3}$ \\ ${ }^{1}$ Nanophotonics Research Laboratory, Department of Electrical and Electronics Engineering, \\ TOBB University of Economics and Technology, Ankara 06560, Turkey \\ ${ }^{2}$ Electrical and Electronics Engineering Department, Ankara University, Gölbasi, Ankara 06830, Turkey \\ ${ }^{3}$ Department of Electrical and Electronics Engineering, Bilkent University, Bilkent, Ankara 06800, Turkey
}

(Received 20 November 2013; accepted 27 December 2013; published online 23 January 2014)

\begin{abstract}
To provide asymmetric propagation of light, we propose a graded index photonic crystal (GRIN PC) based waveguide configuration that is formed by introducing line and point defects as well as intentional perturbations inside the structure. The designed system utilizes isotropic materials and is purely reciprocal, linear, and time-independent, since neither magneto-optical materials are used nor time-reversal symmetry is broken. The numerical results show that the proposed scheme based on the spatial-inversion symmetry breaking has different forward (with a peak value of 49.8\%) and backward transmissions $(4.11 \%$ at most) as well as relatively small round-trip transmission (at most $7.11 \%$ ) in a large operational bandwidth of $52.6 \mathrm{~nm}$. The signal contrast ratio of the designed configuration is above 0.80 in the telecom wavelengths of $1523.5-1576.1 \mathrm{~nm}$. An experimental measurement is also conducted in the microwave regime: A strong asymmetric propagation characteristic is observed within the frequency interval of $12.8 \mathrm{GHz}-13.3 \mathrm{GHz}$. The numerical and experimental results confirm the asymmetric transmission behavior of the proposed GRIN PC waveguide. (C) 2014 AIP Publishing LLC. [http://dx.doi.org/10.1063/1.4861926]
\end{abstract}

Diode-like optical devices play a key role in electronic and optical systems especially for the rectification of excited signals to control the power level and isolation of circuit designs from undesired signals. By definition, an electrical diode is a two-terminal and non-linear device that tends to conduct the current to flow only when it is forward-biased and in the case of reverse-biased, it blocks the current, i.e., it rectifies the current to flow unidirectionally. In a similar manner, an optical isolator (diode) may be defined as an active or passive device that allows an incident light to pass through the structure only in one direction. Such devices that enable unidirectional propagation have received great attention in the fields of optics and photonics. To date, different approaches have been applied to achieve optical isolation, including the use of nonlinear, ${ }^{1}$ time-dependent, ${ }^{2}$ or magneto-optical ${ }^{3}$ structures. That type of design activates optical non-linearity and breaks time-reversal inversion symmetry, thereby breaking reciprocity to attain an adequate optical isolation. Recently, several studies have been conducted in order to accomplish asymmetric light transport by using passive and linear structures such as photonic crystals (PCs) based on the principle of spatial-inversion symmetry breaking. ${ }^{4-7}$ Wang et al. achieved asymmetric wave propagation by Si-based PC heterostructures. ${ }^{8}$ The proposed configuration is linear, passive, and time-independent albeit having broken spatial inversion symmetry. In other words, the structural geometry of the proposed scheme is not symmetrical in terms of mid-point along the $x$-direction, which breaks the spatial symmetry. $21.3 \%$ of forward transmission at most is obtained, and the reported contrast ratio is 0.885

\footnotetext{
${ }^{\text {a) }}$ Author to whom correspondence should be addressed. Electronic mail: igiden@etu.edu.tr
}

as a peak value. Even though the proposed scheme in Ref. 4 was considered to be an optical isolator, according to the comment of Petrov et al., ${ }^{9}$ a reciprocal device having symmetric dielectric permittivity and magnetic permeability tensors cannot enable an optical isolation property, since it possesses a symmetric scattering matrix.

In this paper, a photonic waveguide structure having a graded index profile is presented to achieve an asymmetric light propagation. The waveguide design is realized by introducing point- and line-defects inside rectangular-lattice PCs having a gradually varying index distribution along the longitudinal $x$-direction. Figure 1 (a) shows the graded index PC (GRIN PC) design without any defects and Fig. 1(b) demonstrates the corresponding stair-step refractive index profile. The radii of PC rods are $r=0.20 a$, where $a$ is the lattice constant, and the relevant refractive index equals 3.13 . The design of the GRIN PC structure is achieved by relocating the positions of the PC dielectric rods. Continuously graded refractive index media can be approximated by changing the locations of the PC dielectric rods with a fixed refractive index. To obtain approximately a desired index profile within certain wavelengths, one can just change the spacing of adjacent rods in a predefined manner. The spatial distributions of PC cells are modulated in accordance with a hyperbolicsecant index profile, which is formulated by the following equation, $n(x)=n_{0} \operatorname{sech}(\alpha x)$. In this statement, $\alpha$ is the gradient factor and $n_{0}$ is the effective refractive index at $x=0$, i.e., at the left surface of the proposed structure. The constant $\alpha$ parameter is taken to be $0.0373 a^{-1}$. The effective refractive indices at $x=0$ and $16 a$ are equal to $n(x=0)=n_{0}=2.20$ and $n(x=L=16 a)=1.41$, respectively. While the distance between each rod is modified to capture the targeted longitudinal index distribution, the lateral spacing is fixed at $a$. The 
(a)

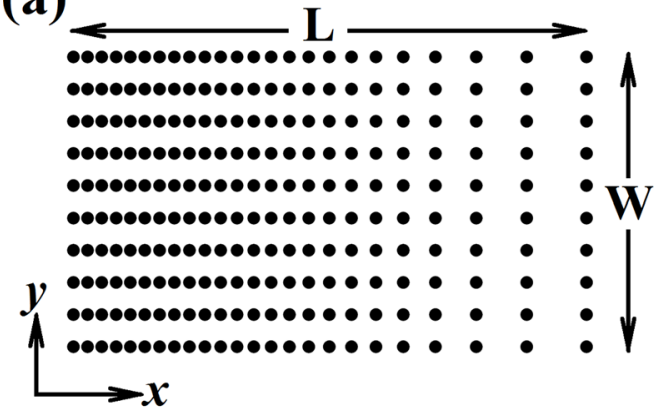

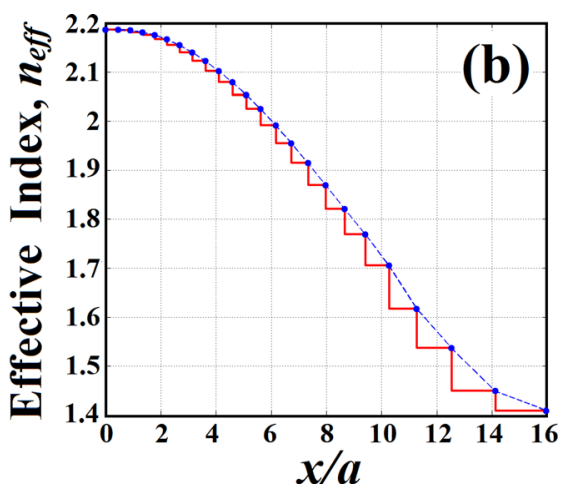

FIG. 1. (a) Schematic view of GRIN PC configuration with its geometrical parameters and (b) the corresponding stair-step effective refractive index profile along the $x$-direction. detailed spectral investigation of the GRIN PC media having such an index profile is analyzed in Ref. 10. The lateral length of the structure is $W=20 a$. The proposed configuration is reciprocal, i.e., linear and time-invariant, since transparent materials (Alumina rods, $\mathrm{Al}_{2} \mathrm{O}_{3}$ ) are utilized. Thus, the system does not include any intrinsic material losses.

At the initial design stage, a line-defect is introduced inside the GRIN PC structure in Fig. 1(a) in order to investigate guided mode (see Fig. 2(a)). The finite-difference timedomain method (FDTD) ${ }^{11}$ is carried out for asymmetric wave propagation performance analyses of the proposed PC structure with the grid sizes of $d x=d y=a / 30$. It should be noted that the studied PC configuration is two-dimensional and the third $z$-dimension is taken to be uniform. The computational window is surrounded by perfectly matched layers, whose thickness is adjusted to 2.0 a . Through all the numerical calculations, transverse magnetic (TM) mode is implemented and then, the concerning non-zero field components are $E_{z}, H_{x}$, and $H_{y}$. Figure 2(a) represents the GRIN PC based waveguide configuration along with the definitions of forward and backward directions. The transmission and signal contrast ratio spectra are shown in Fig. 2(b). The waveguide structure in Fig. 2(a) is excited with a broad Gaussian source located in one side and a detector is placed on the other side in order to measure the output power flux. Then, the forward (the excitation is at the left-side) and backward (the excitation is at the right-side) transmissions are obtained by normalizing the measured flux with that for the incidence case. The calculated normalized transmission values for the forward and backward cases reach 0.0726 (7.26\%) and 0.248 $(24.8 \%)$ at the peak, respectively. Besides for the forward and backward transmission spectra, Fig. 2(b) demonstrates the signal contrast ratio $C_{b f}$, which is defined by
$C_{b f}=\left(T_{b}-T_{f}\right) /\left(T_{b}+T_{f}\right)$. In that expression, $T_{f}$ represents the forward normalized transmission and $T_{b}$ shows the corresponding backward transmission. The regarding signal contrast ratio $C_{b f}$ is in the range of $0.412-0.773$ within the operating frequency band. Such a difference between forward and backward excitation cases and a relatively high contrast ratio imply that asymmetric light transport can be achieved by a GRIN PC based waveguide configuration that possesses a gradually varying index profile along the light propagation direction.

In the next design process, point-defects are intentionally introduced to the waveguide structure to increase the difference between left-to-right (forward) and right-to-left (backward) transmissions. The waveguide structure incurs remarkable lateral losses due to light leakages through the large separations between adjacent rods along the right-side (backward side). When an incident beam encounters the device, there also occur further losses due to back-reflections at the PC-air interface. The inserted point-defects suppress the leakage light at the adjacent rod separations at the right-side so that right-to-left light transmission strengthens. The introduced point-defects are given and shown by dashed boxes in Fig. 3(a). Moreover, since the neighboring rods to the linedefect affect the guided modes of the PC structure, a sequential optimization is pursued on the positions of adjacent rods by introducing shifts $\pm \Delta y$ along the $y$-direction. By this means, we expect that the asymmetric transmission inside the optimized structure could be enhanced. The final configuration of the GRIN PC waveguide through a specific optimization of the neighboring rods' locations is shown in Fig. 3(a) and the optimized parts of PC rods are zoomed in the same figure. The corresponding forward/backward transmission and signal contrast ratio are represented in Fig. 3(b).
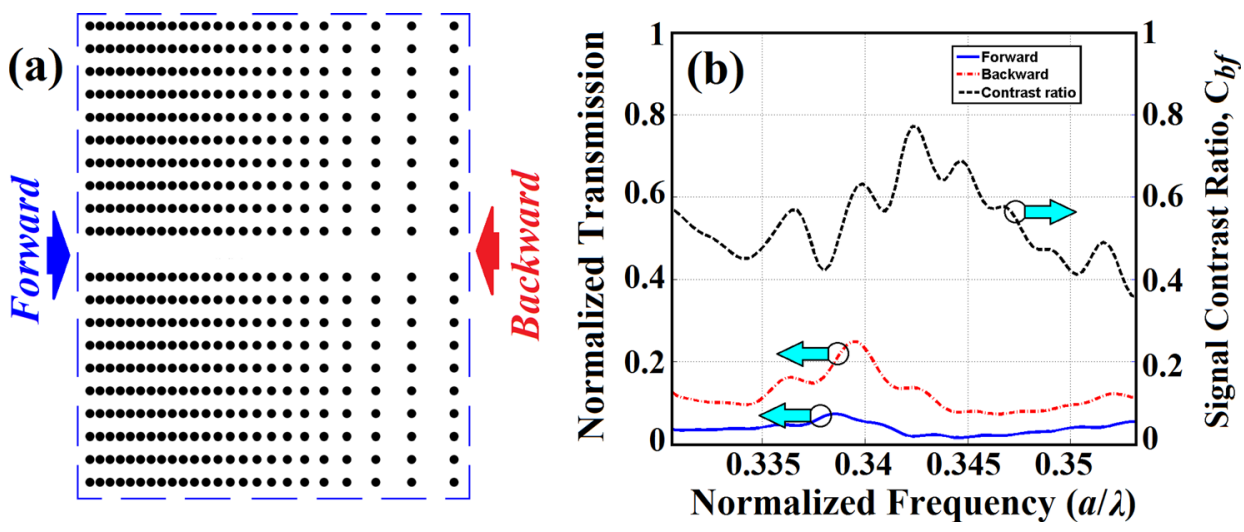

FIG. 2. (a) Schematic of the GRIN PC based waveguide structure with a linedefect. (b) The regarding forward, backward transmission, and signal contrast ratio spectra. 


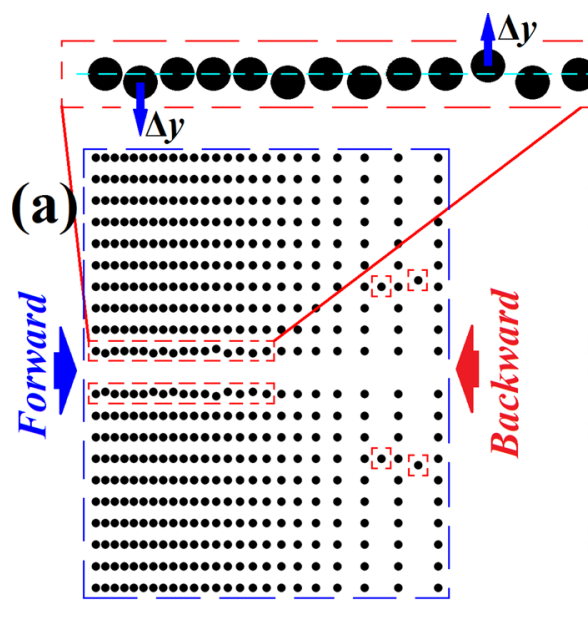

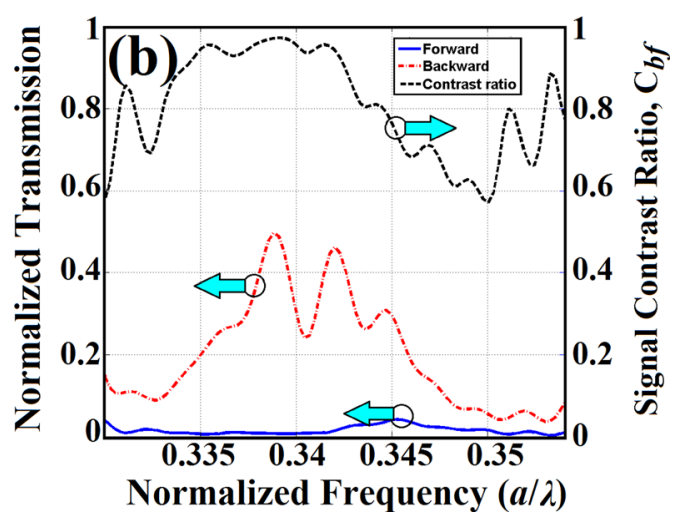

FIG. 3. (a) Schematic view of the optimized GRIN PC based waveguide structure by intentionally introduced line- and point-defects. The pointdefects inside the PC configuration are covered by dashed boxes and the zoomed view of perturbed PC rods is also depicted in the same figure. (b) The forward, backward transmission, and signal contrast ratio spectra for the optimized configuration are shown in (a).
Compared to the results of non-optimized structure case in Fig. 2(a), the peak of the forward transmission $T_{f}$ is reduced from $7.26 \%$ to $4.11 \%$, and the highest backward transmission value $T_{b}$ is increased from $24.8 \%$ to $49.8 \%$. The regarding contrast ratio $C_{b f}$ has a peak value of 0.974 , as it is seen from Fig. 3(b). Within the frequency range of $a / \lambda=0.3332-0.3447$, the corresponding signal contrast ratio is higher than 0.80 and in the frequency interval of $a / \lambda=0.3350-0.3452$, the calculated backward transmission is above $20 \%$. While setting the center frequency at $f_{c e n}=0.3388(a / \lambda)$, the regarding lattice constant turns out to be $a=525.14 \mathrm{~nm}$ to operate in telecom wavelengths. In that case, the optimized structure has a significant asymmetric transmission characteristic in the wavelength range of $1523.5-1576.1 \mathrm{~nm}$ and that, in turn, means an operating bandwidth of $52.6 \mathrm{~nm}$. Strictly speaking, the GRIN PC waveguide structure without any point-defects or artificial perturbations inside the structure may resemble a chirped PC waveguide. ${ }^{7}$ However, the numerically and experimentally investigated forward and backward transmission results were small and the operational bandwidth was narrow.

Figures 4(a) and 4(b) present the time domain snapshots of beam propagations for leftward and rightward cases. The corresponding field patterns are extracted at the operating frequency of $f_{c e n}=0.3388(\mathrm{a} / \lambda)$, since the highest backward transmission is observed at that frequency. To check whether a good asymmetric light propagation is succeeded, we also calculate the round-trip transmission of incident light for an inversely cascaded structure, whose transmission spectrum is represented in Fig. 4(c). It should be reminded that if the round-trip transmission is relatively smaller than the forward transmission, then a good asymmetric wave transport property can be expected from the configuration. ${ }^{4}$ In this study, the single GRIN PC structure is implemented to calculate the forward and backward transmissions, whereas the inversely cascaded configuration is used for the analyses of round-trip transmission. Figure 4(c) collects the forward/backward transmission spectra for the single optimized waveguide as shown in Fig. 3(a) and the regarding round-trip transmission of the inversely cascaded GRIN PC configuration. As shown in Fig. 4(c), the peak value of round-trip transmission is at most $7.0 \%$, which is much smaller than the maximum forward transmission value of $49.8 \%$. The calculated spectra in Fig. 4(c) clarify that the proposed scheme possesses a considerable asymmetric propagation characteristic.

Experimental verification is also carried out in the microwave regime to investigate the asymmetric propagation behavior of a designed 2D PC waveguide. The experimental setup includes an Anritsu two-port 37369A Network Analyzer and two standard pyramidal Ku-band horn
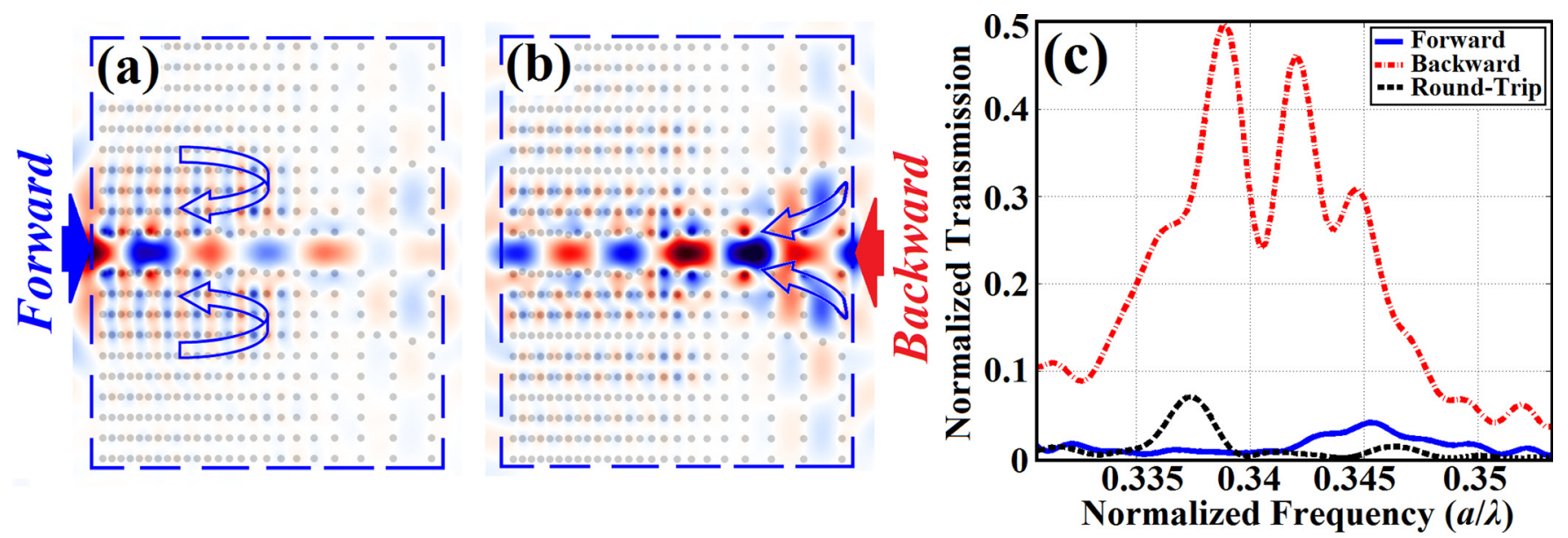

FIG. 4. Time-domain snapshots of the optimized structure in Fig. 3(a) for (a) forward incidence and (b) backward incidence cases. Arrows inserted in the figures (a) and (b) represents the followed paths of the beam that propagates inside PCs. (c) The corresponding forward/backward transmissions for a single and round-trip transmission spectrum for inversely cascaded structure. 
antennas that operate in the frequency range of 12 $\mathrm{GHz}-18 \mathrm{GHz}$. The corresponding measurements are presented in Fig. 5. The studied structure is composed of cylindrical Alumina rods with a refractive index of $n=3.13$ and a diameter of $d=3.1 \mathrm{~mm}$. The regarding lattice constant is then set to $a=7.75 \mathrm{~mm}$. The height of the dielectric rods is so long (longer than $6.5 \lambda$ at $12.8 \mathrm{GHz}$ ) that the illuminated wave propagates inside the structure without suffering from considerable out-of-plane losses. The measured forward/backward transmission spectra and the signal contrast ratio depending on the transmission measurements are represented in Fig. 5. According to the experimental results, the studied PC waveguide design possesses a tolerable contrast ratio value exceeding 0.80 and reaching up to $C_{b f}=0.89$. There is a strong asymmetric propagation characteristic within the frequency interval of $12.8-13.3 \mathrm{GHz}$. Especially, at around operating frequency of $13.11 \mathrm{GHz}$, the forward propagation is below $5 \%$ and backward propagation stays above $50 \%$. We should emphasize the fact that such a high asymmetric transmission characteristic is validated via microwave experimental set up. The measurements are also compatible with the calculated results in 2D FDTD analysis where the corresponding frequency interval was $12.89-13.34 \mathrm{GHz}$ (in the normalized range of $a / \lambda=0.3332-0.3447$ ) and the peak transmission values for forward/backward cases were $4.11 / 49.8 \%$. Scaling up the frequency to optical regime necessitates the deployment of micron-size photonic crystal structure in the experiment with appropriate light sources and detectors.

To thoroughly examine the physical meaning of asymmetric light propagation in linear and passive GRIN PC based devices, the proposed structure is assumed to have three channels: two longitudinal (input/output) channels are adjusted to the input and output waveguide channels along the propagation direction, whereas the third one is considered to describe leakages as well as scattering power along the other directions. Thus, although the total power at all channels is conserved as the system is lossless and time-reversible, ${ }^{12}$ there may occur differences in terms of transmitted power between the input and output channels due to variations on the scattering and leakage powers along the transverse ( $y$-plane) side channels for the forward/backward excitations. That difference in power-level confirms the use of reciprocal devices for asymmetric light propagation.

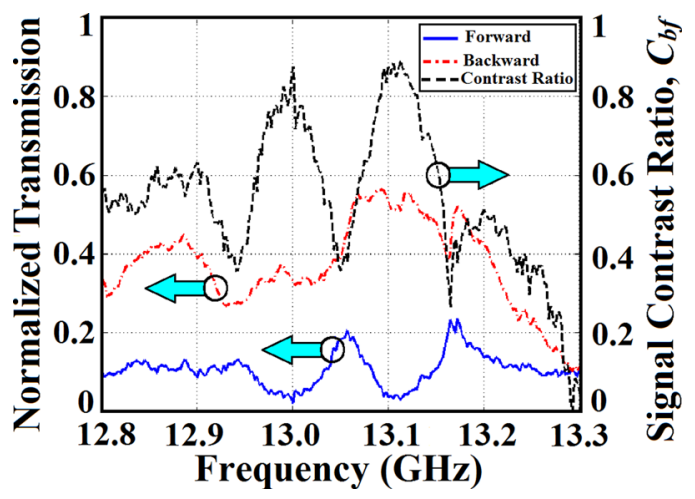

FIG. 5. Experimental measurements of forward and backward transmitted microwave signals. The contrast ratio is also plotted in the same figure.
Another way to the explanation of asymmetric light propagation can be based on the scattering matrix theory. According to the scattering matrix approach adopted in Ref. 13, total power should be conserved in a lossless system. Then, the scattering matrix $S$ should satisfy the unitary property of matrices condition, $S^{-1}=S^{+}$, where $S^{+}$is the conjugate transpose of $S$. Moreover, since the reciprocal systems are time-reversible, then, the inverse of the scattering matrix should be equal to its complex conjugate, that is $S^{-1}=S^{*}$. These two properties implies that the scattering matrix $S$ is symmetric and hence, $s_{i j}=s_{j i}$. To be more specific, the designed GRIN PC system is considered to have three channels: A (forward), B (backward), and C (leakage and scattering powers). Therefore, the relationship between input and output powers from the channels as well as the regarding scattering matrix should be like the following:

$$
\left[\begin{array}{l}
A_{\text {out }} \\
B_{\text {out }} \\
C_{\text {out }}
\end{array}\right]=S\left[\begin{array}{l}
A_{\text {in }} \\
B_{\text {in }} \\
C_{\text {in }}
\end{array}\right], \text { and } S=\left[\begin{array}{lll}
s_{11} & s_{12} & s_{13} \\
s_{12} & s_{22} & s_{23} \\
s_{12} & s_{23} & s_{33}
\end{array}\right],
$$

since the scattering matrix $S$ is symmetric. According to Eq. (1), for the forward-illumination case, i.e., arranging the input signals as $A_{i n}=1, B_{i n}=0$, and $C_{i n}=0$, the output power from channel $\mathrm{C}$ equals $C_{\text {out }}=s_{13}$. However, for the backward-incidence case, i.e., input signals set to $A_{\text {in }}=0$, $B_{\text {in }}=1$, and $C_{i n}=0$, then, the output power from channel $\mathrm{C}$ is equal to $C_{\text {out }}=s_{23}$. Even though our designed structure is a multimode waveguide and each mode at the channels should be labeled as different ports, ${ }^{9}$ obtaining different output powers from channel $\mathrm{C}$ in the forward and backward incidence cases may claim that an asymmetric light propagation can be obtained by the use of linear and passive devices.

In conclusion, the numerical calculations show that the proposed GRIN PC waveguide structure displays a strong asymmetric light propagation characteristic that is in accordance with the reciprocal principle for a linear and timeindependent structure. The optimized configuration, having intentional perturbations and point-/line-defects, exhibits a large difference between the forward $(49.8 \%$ at most) and backward $(4.11 \%$ at the peak) transmissions. The corresponding signal contrast ratio is around 0.80 within the wavelength range of 1523.5-1576.1 nm with an applicable bandwidth value of $52.6 \mathrm{~nm}$. According to the experimental results in microwave regime, the studied GRIN PC waveguide possesses a strong asymmetric propagation characteristic within the frequency interval of $12.8-13.3 \mathrm{GHz}$. Moreover, since only isotropic materials are utilized in the designed structure, it may be implemented practically for the asymmetric wave propagation applications in photonic integrated circuits.

The first four authors gratefully acknowledge the financial support of the Scientific and Technological Research Council of Turkey (TUBITAK), project 110T306. In addition, D.Y. acknowledges the financial support from TUBITAK-BIDEB and H.K. also acknowledges partial support from the Turkish Academy of Sciences. 
${ }^{1}$ R. Philip, M. Anija, C. S. Yelleswarapu, and D. V. G. L. N. Rao, Appl. Phys. Lett. 91, 141118 (2007).

${ }^{2}$ Z. Yu and S. Fan, Nat. Photonics 3, 91 (2009).

${ }^{3}$ A. Figotin and I. Vitebsky, Phys. Rev. E 63, 066609 (2001).

${ }^{4}$ C. Wang, X. Zhong, and Z. Li, "Linear and passive silicon optical isolator," Sci. Rep. 2, 674 (2012).

${ }^{5}$ A. Cicek, M. B. Yucel, O. A. Kaya, and B. Ulug, Opt. Lett. 37, 2937 (2012).

${ }^{6}$ V. Liu, D. A. B. Miller, and S. Fan, Opt. Express 20, 28388 (2012).

${ }^{7}$ H. Kurt, D. Yilmaz, A. E. Akosman, and E. Ozbay, Opt. Express 20, 20635 (2012).

${ }^{8}$ C. Wang, C. Z. Zhou, and Z. Y. Li, Opt. Express 19, 26948 (2011).
${ }^{9}$ A. Petrov, D. Jalas, M. Eich, W. Freude, S. Fan, Z. Yu, R. Baets, M. Popović, A. Melloni, J. D. Joannopoulos, M. Vanwolleghem, C. R. Doerr, and H. Renner, e-print arXiv:1301.7243, Comment on "Linear and passive silicon optical isolator" in Scientific Reports 2, 674 (2013).

${ }^{10}$ H. Kurt, B. B. Oner, M. Turduev, and I. H. Giden, Opt. Express 20, 22018 (2012).

${ }^{11}$ F. Oskooi, D. Roundy, M. Ibanescu, P. Bermel, J. D. Joannopoulos, and S. G. Johnson, Comput. Phys. Commun. 181, 687 (2010).

${ }^{12} \mathrm{H}$. A. Haus, Waves and Fields in Optoelectronics (Prentice-Hall, Englewood Cliffs, NJ, 1984).

${ }^{13}$ A. A. Maznev, A. G. Every, and O. B. Wright, Wave Motion 50, 776-784 (2013). 